ज्) FRANÇAISE

$>\mathrm{DE}$

딜 PÉDAGOGIE

\section{Revue française de pédagogie}

Recherches en éducation

178 | janvier-mars 2012

Les politiques de lutte contre les inégalités scolaires d'un pays à l'autre

\title{
L'utilisation de la vidéo dans la formation professionnelle des enseignants novices
}

Video use in beginning teachers' professional development

La utilización del vídeo en la formación profesional de los docentes principiantes Anwendung der Videoressourcen in der Grundausbildung angehender Lehrer

Cyrille Gaudin et Sébastien Chaliès

\section{(2) OpenEdition \\ Journals}

Édition électronique

URL : http://journals.openedition.org/rfp/3590

DOI : $10.4000 / \mathrm{rfp} .3590$

ISSN : 2105-2913

Éditeur

ENS Éditions

Édition imprimée

Date de publication : 15 mars 2012

Pagination : 115-130

ISBN : 978-2-84788-372-5

ISSN : 0556-7807

Distribution électronique Cairn

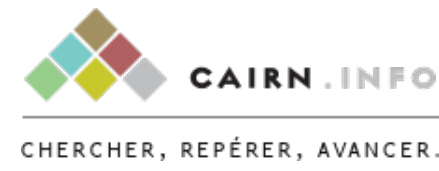

Référence électronique

Cyrille Gaudin et Sébastien Chaliès, «L'utilisation de la vidéo dans la formation professionnelle des enseignants novices », Revue française de pédagogie [En ligne], 178 | janvier-mars 2012, mis en ligne le 15 mars 2015, consulté le 06 mai 2019. URL : http://journals.openedition.org/rfp/3590 ; DOI :

$10.4000 /$ rfp.3590 


\section{L'utilisation de la vidéo dans la formation professionnelle des enseignants novices Cyrille Gaudin et Sébastien Chaliès}

On constate une utilisation accrue de la vidéo dans la formation professionnelle des enseignants novices. Due notamment à la réforme institutionnelle de cette dernière, cette intégration de la vidéo vise le renforcement des liens entre la formation à l'université et les stages en établissement scolaire. La vidéo apparaît en effet comme un moyen privilégié pour favoriser cette alternance en raison de sa capacité unique à capturer la richesse et la complexité des salles de classe. Si son utilisation dans la formation professionnelle des enseignants novices semble apparemment faire consensus, une lecture fine de la littérature du domaine laisse toutefois entrevoir des points d'accord, des controverses mais aussi des "zones potentielles d'étude ". Sur la base d'une explicitation de ces principales zones, cet article invite finalement à engager une réflexion sur les circonstances dans lesquelles l'usage de la vidéo peut constituer un trait d'union entre le caractère virtuel de l'enseignement en formation et sa réalité dans le contexte de la classe.

Mots-clés (TESE) : formation initiale des enseignants, formation en alternance, apprentissage en ligne, enseignant stagiaire, TIC.

Cet article porte sur l'utilisation de la vidéo dans les situations de formation professionnelle des enseignants novices (EN par la suite). Trouvant sa justification dans la préoccupation sociale de la formation des EN et la multiplicité des travaux menés dans le domaine, cet article a pour objet de synthétiser la littérature nationale et internationale du domaine, de délimiter le champ des travaux qu'il reste à mener dans le domaine et enfin de rendre possible la construction et la mise en œuvre par les formateurs de dispositifs utilisant la vidéo. Pour ce faire, il est organisé en trois parties. La première partie montre que la multiplication des situations de formation professionnelle utilisant la vidéo pour observer et analyser le travail enseignant est une des conséquences de la mise en place de dispositifs de formation fondés sur le principe de l'alternance. La deuxième partie détaille plus spécifiquement les principales "zones potentielles d'études » ouvertes suite à la lecture minutieuse de la littérature relative à l'utilisation de la vidéo en formation professionnelle des EN. Pour conclure, la dernière partie montre qu'une réflexion est nécessaire pour 
optimiser l'utilisation de la vidéo afin qu'elle contribue à minimiser la différence existant entre le caractère virtuel de la formation et les circonstances réelles d'intervention en classe.

\section{L'UTILITÉ DE LA VIDÉO DANS LES DISPOSITIFS DE FORMATION DES ENSEIGNANTS NOVICES}

Ces dernières années, une dynamique institutionnelle en faveur d'un développement de la dimension professionnelle des formations adressées aux futurs enseignants au sein des universités est à noter (Escalié \& Chaliès, 2011). Pour soutenir cette dynamique, certains textes officiels invitent à la mise en place de dispositifs de formation fondés sur le principe d'une alternance effective entre l'université et les établissements scolaires. La commission des communautés européennes mentionne par exemple que " les établissements de l'enseignement supérieur ont un rôle majeur à jouer dans la création de partenariats efficaces avec les écoles et les autres acteurs pour veiller à ce que leurs programmes de formation pour enseignants reposent sur des faits concrets et établis et sur une bonne expérience des salles de classe " (Commission des communautés européennes, 2007, p. 16). Dans le prolongement de cette recommandation européenne, la réforme de la formation des enseignants engagée en France (MESR, 2008) invite aussi à davantage de relations entre les différentes institutions que sont les universités et les établissements scolaires, engagés dans la formation professionnelle des EN. Dans le même ordre d'idées, la circulaire du 23 décembre 2009 relative à la mise en place des diplômes nationaux de master ouverts aux étudiants se destinant aux métiers de l'enseignement stipule que " les masters doivent intégrer une composante forte de formation professionnelle basée sur un "aller-retour" entre pratique du métier et formation à l'université " (MESR, 2010, p. 3). Une des conséquences de la mise en place de dispositifs de formation fondés sur ce principe de l'alternance est la multiplication des situations de formation professionnelle basées sur l'observation et l'analyse du travail enseignant. Le récent recours à la technologie, et notamment à la vidéo, s'inscrit généralement dans cette nouvelle dynamique institutionnelle. Toutefois, il peut aussi être interprété comme un palliatif de la disparition de certains pans de la formation professionnelle (en particulier la diminution du nombre et de la diversité des stages proposés tout au long du cursus de formation des futurs enseignants). C'est par exemple le cas de modalités de formation récemment apparues dans le contexte français et soutenues par le ministère de l'Éducation nationale. La plateforme de formation " tenue de classe ${ }^{1}$ " préconise par exemple des attendus académiques en prescrivant des conduites à tenir, alors que la plateforme “ Néopass@ction² »s'appuie sur des travaux de recherche portant sur le travail réel des enseignants novices pour décrire leurs stratégies, les dilemmes et les compromis provisoires qu'ils se donnent au quotidien.

Alors que la salle de classe reste le contexte d'observation et d'analyse du travail par excellence (Postic, 1977 ; Putnam \& Borko, 2000), les situations de formation des EN ne peuvent pas effectivement s'y restreindre (Borko, Jacobs, Eiteljorg et al., 2008). Les documents pédagogiques de l'enseignant en activité, les réalisations des élèves, mais aussi et surtout les enregistrements vidéo des leçons (Ball \& Cohen, 1999) sont ainsi autant de traces pouvant être rapatriées dans les situations de formation mises en œuvre à l'université pour mieux observer ce qui s'est passé en classe, l'analyser et par là-même se former au métier (Carter, 1999). Exploité dans de nombreux pays, l'enregistrement vidéo apparaît toutefois dans la littérature comme 
" un artefact de choix de la pratique d'enseignement en classe » (Borko, Jacobs, Eiteljorg et al., 2008 ; Koc, 2011 ; Santagata, 2009), et ceci pour deux raisons principales. Tout d'abord, l'enregistrement vidéo est un artefact efficace pour « plonger la formation initiale des enseignants dans les questions, les problèmes et les solutions d'enseignement » (Richardson \& Kile, 1999, p. 122), c'est-à-dire pour rompre avec de nombreuses séquences de formation réalisées à l'université et centrées sur des apports exclusivement théoriques (Koehler, 2002 ; Richardson \& Kile, 1999 ; Shulman, 1992). II permet, d'autre part, de placer les EN dans une situation privilégiée pour observer et analyser la complexité des événements survenus en classe (Brophy, 2004). Pour Sherin (2004), l'enregistrement vidéo est en effet un artefact qui permet " d'entrer dans le monde de la salle de classe " sans toutefois être contraint d'y enseigner. II place plus exactement les EN au cœur de situations de formation fictionnelles (Durand, 2008 ; Schaeffer, 1999) dans lesquelles ces derniers peuvent s'exercer à " penser comme un enseignant » (Kleinfeld, 1988) et à " procéder par procuration à des anticipations " (Ria, Serres \& Leblanc, 2010), tout en restant épargnés par la complexité et l'imprévisibilité de la salle de classe. II est toutefois à noter que, bien que les progrès technologiques récents (en termes notamment de numérisation de la vidéo et de développement de logiciels et d'outils en ligne) aient contribué à l'augmentation du recours à la vidéo pour étayer le développement professionnel des EN (Brunvand, 2010 ; Goldman, 2007 ; Hiebert, Gallimore \& Stigler, 2002 ; Krammer, Ratzka, Klieme et al., 2006), cette dernière n'est toutefois pas récente dans le cadre plus large de leur formation professionnelle. Dès les années soixante-dix, les pratiques dites de "vidéo-formation "s'inscrivent principalement dans une approche objectiviste de la situation d'enseignement. Elles ont, en ce sens, contribué à l'aménagement des plans de formation des EN grâce notamment à la mise en œuvre de nouvelles situations d'observation de l'activité professionnelle, d'introspection, de micro-enseignement ou d'essais pédagogiques (Mottet, 1996).

En apparence, un consensus tant institutionnel que scientifique peut donc être relevé quant à l'utilité de la vidéo dans les dispositifs de formation professionnelle des EN basés sur le principe de l'alternance entre l'université et les établissements scolaires. Une lecture fine de la littérature nationale et internationale permet toutefois de circonscrire plusieurs champs de recherche que nous assimilerons à des "zones potentielles d'études " et que nous justifierons par la suite. Tel que le précise fort justement Sherin (2004, p. 10), « il semble que la vidéo soit devenue un élément permanent dans la formation des enseignants. Ce qui est surprenant, cependant, c'est que, malgré son ampleur, l'utilisation de la vidéo dans la formation des enseignants ne reflète pas toujours la compréhension précise de ce que la vidéo pourrait fournir dans l'apprentissage des enseignants ". Par la suite, nous nous efforçons donc de délimiter successivement différentes possibilités d'étude dans le domaine pour parvenir, à terme, à une meilleure compréhension des effets de la vidéo dans la formation des EN et à une construction optimisée des situations en faisant usage.

\section{LES PRINCIPALES ZONES POTENTIELLES D'ÉTUDE CONCERNANT L'UTILISATION DE LA VIDÉO DANS LA FORMATION DES ENSEIGNANTS NOVICES}

\section{L'activité des enseignants novices au cœur des situations de formation utilisant la vidéo}

De nombreuses études relèvent que les EN rencontrent des difficultés à observer et à analyser efficacement les situations d'enseignement-apprentissage qui leur sont 
présentées ou qu'ils mettent en œuvre lorsqu'ils sont en classe (Gaudin \& Chaliès, 2011a ; Girard, 1999 ; Putnam \& Borko, 2000). Ils ne parviennent pas, par exemple, à observer et analyser in situ les principaux « organisateurs » (Bru, Pastré \& Vinatier, 2007) de chacune des situations se déroulant sous leurs yeux, alors même que les enseignants expérimentés parviennent quant à eux à s'en saisir rapidement (Frederiksen, 1992 ; Jacobs \& Morita, 2002 ; Leinhardt, Putnam, Stein et al., 1991; van Es \& Sherin, 2008). Les résultats de l'étude de Delpoux et Veyrunes (2010), menée auprès de professeurs des écoles novices en stage d'observation montrent par exemple que leur activité s'apparente à du " butinage ", c'est-à-dire à une analyse " en surface " de l'activité de l'enseignant et des élèves. Le caractère interdépendant des comportements de ces derniers n'est par exemple que très rarement accessible aux EN lorsqu'ils les observent. Dans le même ordre d'idées, le caractère pluridimensionnel (temporel, spatial, les placements, le matériel exploité, la nature des consignes, etc.) du contexte de la classe est régulièrement atrophié au bénéfice d'une centration exclusive sur l'une de ces dimensions (par exemple le placement de l'enseignant).

Sur la base de ce constat, de nombreuses études ont proposé d'utiliser la vidéo afin de mieux accompagner les EN dans la construction de leur capacité à observer et à attribuer une signification aux événements de la classe. Paradoxalement, le constat relatif à la " cécité " des EN face aux situations d'enseignement-apprentissage n'en est pas moins resté toujours aussi prégnant (Sweller, 1994, 2004). Certaines études récentes ont par exemple mis en exergue la difficulté pour les EN à faire preuve de discernement lorsqu'ils visionnent un enregistrement vidéo d'une discipline qu'ils ne connaissent pas (Jacobs, Lamb, Philipp et al., 2007 ; Star \& Strickland, 2008 ; van Es \& Sherin, 2008). Ils se concentrent alors sur des " éléments superficiels " tels que les caractéristiques des enseignants ou des élèves, les problèmes inhérents à la gestion de la classe, et ne parviennent pas à s'extraire de « jugements globaux sur l'efficacité de la leçon " (Castro, Clark, Jacobs et al., 2005, p. 11). Ils ne perçoivent en effet qu'un flux continu d'événements sans jamais pouvoir correctement les analyser en temps réel (Erickson, 2007), ce qui au final les maintient dans le rôle de spectateur.

Forts de cette conclusion partagée, les chercheurs du domaine ont emprunté à Goodwin (1994) le concept de "vision professionnelle " et l'ont introduit comme l'une des capacités à construire en cours de formation professionnelle (Sherin, 2001, 2007). Paradoxalement, alors que la compétence à enseigner a été de plus en plus assimilée à la capacité pour les EN à observer les événements les plus pertinents de la classe, à les analyser pour prendre les décisions les plus adaptées et finalement à agir avec pertinence (Borko, Koellner, Jacobs et al., 2011), rares sont encore à ce jour les travaux qui ont étudié leur activité en cours de situation de formation professionnelle utilisant la vidéo. À notre connaissance, seule l'étude de van Es et Sherin (2008), menée auprès de groupes d'EN en mathématiques, investis dans des situations de formation permettant de visionner des enregistrements vidéo et d'en discuter, a interrogé cette dimension. Aidés par un formateur «facilitateur ", les EN parviennent en effet dans ce type de situation à ne plus seulement se focaliser sur l'enseignant et à s'intéresser davantage aux élèves. Ils renforcent d'autre part leur capacité à interpréter et non plus seulement à décrire ce qu'ils observent, grâce notamment à des observations de plus en plus spécifiques au contexte local d'enseignement.

Bien moins étayés que ceux des nombreuses études ayant examiné l'activité effective des enseignants expérimentés lors de l'observation d'enregistrements vidéo (Colestock \& Sherin, 2009 ; Putnam \& Borko, 2000 ; Schwindt, 2008 ; Seidel \& Prenzel, 2007 ; Sherin \& van Es, 2009), les résultats de l'étude de van Es et Sherin 
(2008) permettent malgré tout de délimiter différents modes de construction par les EN de leur capacité à observer et analyser le travail. Pour certains d'entre eux, le mode de construction peut ainsi être qualifié de « direct » : les EN apprennent alors à analyser les événements tout en conservant leur caractère naturellement holistique (chaque événement comprenant les élèves, l'enseignant, le matériel, etc.). Pour d'autres EN, le mode de construction peut être qualifié de «cyclique ": il se caractérise alors par un aller-retour systématique entre des observations générales et d'autres plus spécifiques. Enfin, certains EN semblent engagés dans un mode de construction dit « incrémentiel ou progressif » : à chaque visionnage, chacune de leurs observations s'enrichit de nouvelles dimensions pour finalement aboutir à une analyse quasi exhaustive des événements visionnés. Même si la capacité des EN à observer et analyser les événements de la classe ne se construit certainement pas selon un mode exclusif (Lajoie, 2003), les résultats de cette étude ouvrent toutefois de nouvelles perspectives pour appréhender la complexité de la construction de cette capacité professionnelle (Simon, Tzur, Heinz et al., 2000) et, par voie de conséquence, pour penser autrement l'utilisation de la vidéo en formation. C'est d'ailleurs dans cette optique que van Es et Sherin (2008) proposent en guise de conclusion de leur travail un cadre pour apprendre à observer (learning to notice framework). Selon ces auteurs, cela consisterait finalement pour les EN à être capable d'identifier ce qui est important dans une situation d'enseignement en utilisant ce que l'on sait sur le contexte, mais aussi à établir des liens entre les événements spécifiques de cette situation et des principes plus larges relatifs à l'enseignement et/ou l'apprentissage.

Seulement abordée par certains travaux, l'analyse de l'activité des EN au cours des situations de formation professionnelle utilisant la vidéo apparaît comme un champ de recherche possible (Franke, Carpenter, Fennema et al., 1998 ; HufferdAckles, Fuson \& Sherin, 2004). L'étude de van Es et Sherin (2008) fournit des premiers résultats intéressants et prometteurs. II apparaît toutefois nécessaire d'étudier dans le détail l'activité des EN afin de parvenir à étayer davantage la construction et la mise en œuvre des situations exploitant la vidéo au cœur de dispositifs de formation professionnelle fondés sur le principe de l'alternance.

\section{Modalités d'utilisation de la vidéo et développement professionnel des enseignants novices}

Une des réponses aux difficultés des EN à observer et à analyser les situations d'enseignement-apprentissage a été de multiplier les modalités d'utilisation de la vidéo au sein des situations de formation professionnelle (Borko, Koellner, Jacobs et al., 2011). En prenant appui sur la classification proposée par Merseth (1996), deux grandes modalités, étayées par des fondements théoriques singuliers, peuvent être repérées dans la littérature.

La modalité d'utilisation de la vidéo la plus courante est celle consistant à donner à observer, signifier et analyser une large diversité de pratiques professionnelles (Mayer, Moreno, Boire et al., 1999 ; Brophy, 2004). L'usage de la vidéo s'inscrit alors dans une approche développementaliste de la formation des EN. L'idée n'est pas « d'évaluer des bonnes ou mauvaises pratiques qui seraient à reproduire ou à écarter mais plutôt de permettre de comprendre ces différentes façons de faire comme des formes provisoires d'adaptation à un contexte d'enseignement et à un moment particulier de son développement professionnel » (Leblanc, 2009, p. 6). Dans une perspective située (Lave \& Wenger, 1991), Borko, Jacobs, Eiteljorg et alii (2008) proposent par exemple de présenter aux EN, par l'intermédiaire d'enregistrements vidéo, des exemples de pratiques professionnelles destinés à alimenter non 
pas directement leurs façons de faire, mais plutôt leur démarche d'analyse professionnelle. C'est d'ailleurs en ce sens que ces auteurs proposent des situations de formation collective sous la forme d'un « cycle de résolution de problèmes », comprenant trois ateliers pensés pour successivement permettre aux EN de résoudre des problèmes de mathématiques, d'élaborer des plans pour enseigner une démarche de résolution de ces problèmes, d'étudier la mise en œuvre de ces plans dans leur propre classe à partir d'un enregistrement vidéo, et plus généralement d'étudier la réflexion des élèves à partir de cette vidéo. Dans le même ordre d'idées, Santagata, Zannoni et Stigler (2007) présentent une méthode d'analyse de leçons enregistrées comportant trois étapes centrant successivement les EN sur les objectifs poursuivis au cours de la leçon, sur l'apprentissage des élèves et sur les alternatives possibles en termes d'enseignement. Dans leurs travaux, Zhang, Lundeberg, Koehler et alii (2011) proposent aussi d'exploiter les enregistrements vidéo pour engager des EN de sciences dans une pratique réflexive respectant les principes suivants : demander aux EN d'identifier les aspects pertinents de l'enregistrement vidéo proposé ; mettre l'accent sur leur raisonnement pédagogique en leur demandant d'élaborer des hypothèses qui tiennent compte du problème d'enseignement-apprentissage identifié ; les encourager explicitement à reconnaître leurs propres difficultés professionnelles en comparant leur pratique professionnelle à celle observée en vidéo, et enfin les aider à formuler des commentaires sur la pratique visionnée.

C'est dans cette perspective dite « réflexive » d'exploitation de la vidéo que d'autres études ont proposé des situations de formation de type " micro-enseignement " (Benton-Kupper, 2001 ; Yamamoto \& Hicks, 2007). À ce titre, l'étude récente de Koc (2011), prenant appui sur la théorie du constructivisme social de Vygotski (1978), montre les grandes lignes d'un dispositif de formation exploitant la vidéo pour engager sous la forme de jeux de rôle des EN (de mécanique) dans l'élaboration, la réalisation et l'analyse de séquences d'enseignement. Elle décrit plus précisément la multiplicité des usages de la vidéo au cours des six étapes constituant le dispositif : constitution de groupes d'EN (de 3 ou 4 personnes), identification d'un thème central pour la leçon qui sera collectivement mise en œuvre sous la forme d'un jeu de rôle, réalisation enregistrée de la leçon avec attribution des rôles d'élèves et d'enseignant, montage de l'extrait vidéo support de l'analyse après sélection des événements jugés comme les plus significatifs, analyse collective des événements enregistrés, présentation aux autres EN, discussion collective et écriture d'une réflexion personnelle. En parallèle avec ce type d'utilisation de la vidéo pour engager des collectifs d'EN dans une pratique réflexive, certaines études se sont centrées sur des modalités cherchant à engager, mais aussi à soutenir la réflexion personnelle de chacun des EN (Leblanc, 2009 ; Yakura, 2009). Rodgers (2002) a par exemple élaboré une modalité d'utilisation de la vidéo ordonnée sous la forme d'un cycle de réflexion structuré en trois étapes : les EN décrivent d'abord en détail des situations sélectionnées de leur enseignement, puis les interprètent et enfin décident de remédiations à mettre en œuvre.

La seconde modalité d'utilisation de la vidéo en formation consiste non plus à engager, voire à développer, la pratique réflexive des $\mathrm{EN}$, mais plutôt à leur présenter des pratiques professionnelles exemplaires (Andre, Schmidt, Nonis et al., 2000 ; Goldman \& Barron, 1990). Par cette modalité d'utilisation, la vidéo permet de donner à voir aux EN des exemples de pratiques professionnelles empruntées à des enseignants expérimentés afin d'orienter leur propre façon de faire en classe. En prenant appui sur le modèle de la "flexibilité cognitive " (Jacobson \& Spiro, 1995), Yadav (2008) montre par exemple lors de son étude combien il est enrichissant pour les EN de voir, sous la forme d'extraits vidéo aménagés, la manière dont des enseignants expérimentés parviennent à exploiter différentes stratégies 
d'enseignement considérées comme efficaces, notamment pour les reproduire. Présente de façon significative dans la littérature, cette modalité d'utilisation de la vidéo n'en est pas moins discutée, notamment lorsqu'il s'agit de choisir les exemples donnés à visionner en fonction de leur caractère exemplaire. Positionné dans une épistémologie énactive de l'activité (Varela, 1996), Leblanc (2009, p. 5) note quant à lui qu'« un des enjeux de la formation est d'envisager comment l'activité experte peut être mobilisée en formation, non pas comme une référence unique, mais selon un degré de compatibilité avec l'activité débutante en cours de développement ». Leblanc (2009 ; Leblanc \& Ria, 2010) propose plus précisément en ce sens de dépasser les limites des approches experts/novices qui sont souvent muettes sur les processus de constitution de l'expertise pour plutôt envisager de montrer aux EN les pratiques typiques de leurs pairs, plus proches de leurs préoccupations et par là-même sources de davantage de professionnalisation.

Les modalités d'utilisation de la vidéo dans les situations de formation professionnelle des EN sont donc pensées et/ou étudiées en fonction des postulats théoriques convoqués par les chercheurs (Meloth, Good \& Sugar, 2008). Dans une moindre mesure, elle semble aussi dépendante des objectifs de formation poursuivis dans ces situations (Tochon, 1999). Brophy (2004) précise en effet que pour être un outil efficace dans la formation des enseignants, la vidéo doit être sélectionnée et incorporée à l'ensemble des activités constitutives du programme de formation (par exemple, les stages, les cours magistraux, etc.), compte tenu de l'objectif poursuivi. Pareillement, le Fevre (2004) stipule que « la vidéo n'est pas un curriculum [...]. La vidéo est plutôt un moyen qui peut être développée comme une ressource et utilisée de façon spécifique pour améliorer l'apprentissage [des EN] » (p. 235). Par exemple, dans une étude récente, Sherin et van Es (2009) identifient en effet trois types d'objectifs poursuivis dans les situations de formation des EN exploitant la vidéo. Dans certains dispositifs, la vidéo est principalement utilisée pour aider les EN à apprendre de nouvelles techniques pédagogiques (Bitter \& Hatfield, 1994) et à se préparer à les mettre en œuvre dans leur classe. Dans d'autres dispositifs, elle est exploitée afin de développer les connaissances des EN sur l'enseignement de leur discipline. Elle permet alors une compréhension plus approfondie de la discipline enseignée et des modalités d'apprentissage des élèves (Koellner, Jacobs, Borko et al., 2007). Enfin, dans certaines situations, la vidéo est un support pour aider les EN à mieux analyser et à s'adapter aux événements de la classe (Star \& Strickland, 2008). Largement étudiée, cette multiplicité des modalités d'utilisation de la vidéo dans les situations de formation professionnelle des EN ouvre néanmoins un deuxième champ d'étude dans le domaine. Certains travaux récents invitent en effet à orienter les prochaines études sur des dispositifs s'efforçant d'articuler ces différentes modalités en tenant compte du développement professionnel des EN (voir notamment Sherin et van Es, 2009). Dans le même ordre d'idées, Borko, Koellner, Jacobs et alii (2011) concluent sur la nécessité de travailler à la construction d'un " continuum » dans les modalités d'utilisation de la vidéo, depuis des modalités " hautement adaptatives " valorisant la réflexivité à partir du contexte local d'intervention de l'EN vers des modalités « hautement spécifiées ", plutôt centrées sur l'analyse de cas construits.

\section{La nature des contenus des vidéos utilisées dans les situations de formation}

Alors que les modalités d'utilisation de la vidéo dans la formation des EN sont largement étudiées, peu de travaux ont examiné les contenus donnés à visionner aux EN et leurs effets sur leur formation. Dans leurs grandes lignes, ces quelques travaux montrent que les EN s'approprient par la vidéo les pratiques professionnelles efficaces d'enseignants qu'ils ne connaissent pas (Hatch \& Grossman, 2009). Ils 
montrent aussi que les EN diversifient leurs stratégies d'enseignement et comprennent mieux les raisonnements de leurs élèves lorsqu'ils observent l'enregistrement vidéo de leçons de leurs collègues (Sherin \& Han, 2004). Ils montrent enfin que les EN développent davantage leur réflexivité en observant l'enregistrement vidéo de leurs propres leçons (Rosaen, Lundeberg, Cooper et al., 2008).

Rares sont toutefois les travaux, tels que ceux de Zhang, Lundeberg, Koehler et alii (2011), qui sont allés encore plus loin dans l'investigation en s'efforçant pour un même public d'EN de comparer les effets spécifiques de chaque type de contenu donné à visionner séparément ou simultanément. Les résultats de cette étude montrent que l'utilisation d'enregistrements d'enseignants que les EN ne connaissent pas a pour principale conséquence de fixer un modèle de départ. À partir de ce dernier, les EN vont ensuite pouvoir s'engager dans une analyse réflexive en les comparant avec des enregistrements de leçons réalisées par des collègues ou par eux-mêmes. Ce type d'enregistrement présente néanmoins comme limite de confronter les EN à des contextes d'enseignement souvent bien éloignés de ce qu'ils vivent dans leurs classes, ce qui au final nuit à leur engagement et réduit les possibles exploitations en classe des éléments abordés en formation. Le visionnage d'enregistrements de leçons menées par des collègues a pour principal intérêt de permettre aux EN de sortir de leur isolement au sein de leur établissement scolaire, en accédant à ce que font leurs collègues dans des circonstances d'enseignement proches, voire similaires aux leurs. Les auteurs relèvent que, pour certains EN, des échanges, voire des pratiques de co-enseignement, ont ensuite eu lieu au sein de l'équipe pédagogique. En pouvant « voir l'autre comme soi-même » (Leblanc, 2009), les EN ont en effet pu constater qu'ils étaient confrontés à des problèmes similaires (Borko, Jacobs, Eiteljorg et al., 2008) et ont donc plus facilement accepté de modifier leur pratique professionnelle après avoir pu observer et discuter de nouvelles stratégies d'enseignement. Enfin le visionnage d'enregistrements de leurs propres leçons a pour principal intérêt d'alimenter leur pratique réflexive. Tel « un miroir de leur enseignement » (Zhang, Lundeberg, Koehler et al., 2011), ce type de visionnage leur permet en effet de se placer à distance de leur pratique de classe (notamment par exemple de sa composante émotionnelle), d'apprendre à " se connaître et se reconnaître " (Leblanc, 2009), pour in fine mieux repérer les composantes de leur pratique professionnelle à améliorer (Borko, Jacobs, Eiteljorg et al., 2008). Les EN peuvent en effet, à partir de ce type d'enregistrement, voir des événements passés inaperçus lors de la leçon (Clarke \& Hollingsworth, 2000 ; le Fevre, 2004). Ils peuvent par exemple plus finement observer les effets de leurs interventions auprès des élèves, alors même qu'en cours de leçon ils n'en ont jamais le temps (Borko, Jacobs, Eiteljorg et al., 2008). Dans le même ordre d'idées, ils peuvent visualiser et analyser l'activité des groupes d'élèves (Sherin, 2004).

Tout comme l'étude de Zhang, Lundeberg, Koehler et alii (2011), de récents travaux relèvent que les enregistrements des leçons des EN sont les plus efficaces pour accompagner leur développement professionnel (Borko, Jacobs, Eiteljorg et al., 2008 ; Seidel, Stürmer, Blomberg et al., 2011). Il apparaît en effet que la connaissance du contexte d'enseignement visionné est un des éléments essentiels pour que les EN s'engagent efficacement dans la situation de formation (Goldman, 2007 ; Lemke, 2007 ; Spiro, Collins \& Ramchandran, 2007). Les résultats de l'étude de Seidel, Stürmer, Blomberg et alii (2011) montrent ainsi que, quand les EN observent l'enregistrement vidéo de leurs propres leçons, leur immersion et leur motivation sont nettement supérieures à celles qu'ils ont lorsqu'ils observent des enregistrements ne les concernant pas directement. Ils développent donc davantage leur capacité d'observation, d'analyse et in fine d'action (Krammer, Ratzka, Klieme et al., 2006 ; Sherin \& van Es, 2009 ; Star \& Strickland, 2008). Des études mettent toute- 
fois en avant l'importance de créer une " communauté de soutien » lors de ces situations de formation (Borko, Jacobs, Eiteljorg et al., 2008 ; Grossman, Wineburg \& Woolworth, 2001 ; Sherin \& Han, 2004) : sans cet aménagement, ces derniers peuvent se retrouver en difficulté, voire abandonner la formation, lorsqu'ils sont confrontés à la nécessité d'être filmés et de montrer leur cours à d'autres EN.

Les résultats de ces quelques travaux ayant étudié les effets propres à chaque type d'enregistrement vidéo sur le développement professionnel des EN demandent toutefois à être complétés. D'autres études à venir pourraient chercher à interroger les enchaînements possibles de ces différents types d'enregistrement vidéo au sein des situations de formation. Certains travaux récents appellent en effet dans leur conclusion à l'association des trois types d'enregistrement vidéo décrits plus haut pour former efficacement les EN. Néanmoins, leurs propositions restent à ce jour divergentes. Zhang, Lundeberg, Koehler et alii (2011) proposent par exemple d'utiliser exclusivement les enregistrements vidéo d'enseignants que les EN ne connaissent pas en début de formation pour fixer des «modèles de façon de faire ». Selon ces auteurs, les deux autres types d'enregistrement ne devraient apparaître que dans un deuxième temps de formation. Inversement, Seidel, Stürmer, Blomberg et alii (2011) considèrent qu'il est préférable de débuter la formation des EN en les confrontant à l'enregistrement vidéo de leurs propres leçons. Leblanc (2009) met quant à lui en exergue la nécessité d'instaurer une progressivité dans l'exploitation de ces divers types d'enregistrement vidéo, en s'efforçant de répondre à l'évolution des préoccupations des EN. Pour l'auteur, les situations de type " alloconfrontation ", prenant appui sur les enregistrements vidéo des leçons d'autres enseignants, apparaissent ainsi plus pertinentes à mettre en œuvre en début de formation car elles conservent " une proximité avec l'expérience des EN sans qu'ils soient contraints de l'exposer aux autres ". Les situations de type " autoconfrontation " sont quant à elles à introduire au bout de quelques mois de formation, car " la mise en place de ce travail nécessite une contractualisation avec les acteurs qui est facilitée lorsque les acteurs ont déjà vécu des modalités d'alloconfrontation à l'expérience de pairs " (Leblanc, 2009, p. 13). Enfin notre étude (Gaudin \& Chaliès, 2011a), réalisée dans le cadre d'une théorie de l'action collective (Bertone, Chaliès \& Clot, 2009), montre que l'enchaînement des trois types d'enregistrement vidéo dépend moins de l'évolution des préoccupations des EN que de leurs capacités à analyser les événements à observer. Ces auteurs suggèrent en ce sens que cet enchaînement devrait être pensé pour que les EN puissent constamment voir un « air de famille » entre les différents types d'enregistrement vidéo visionnés.

\section{Les impacts des situations de formation utilisant la vidéo sur la pratique de classe des enseignants novices}

Si la finalité affichée de l'usage de la vidéo lors des situations de formation professionnelle des EN est toujours d'améliorer leur pratique effective de classe, rares sont toutefois les études ayant véritablement cherché à apprécier cet impact. La plupart des études réalisées dans le domaine présupposent en effet, à partir par exemple de réponses à des questionnaires adressés aux EN, qu'il y a des effets de ce type de situation de formation sur l'activité de classe des EN (Kale, Whitehouse \& Hayes, 2009 ; Meloth, 2008 ; Moreno, Abercrombie \& Hushman, 2009) ou plus largement sur leur développement professionnel (Goldman, Pea, Barron et al., 2007). Les preuves empiriques d'effets réels sont à ce jour peu nombreuses (Brophy, 2004 ; Gaudin \& Chaliès, 2010 ; Seidel, Stürmer, Blomberg et al., 2011). C'est avec la volonté de répondre à ce constat que quelques études (Borko, Jacobs, Eiteljorg 
et al., 2008 ; Cohen, 2004 ; Gaudin \& Chaliès, 2011a ; Ria, Serres \& Leblanc, 2010 ; Sherin \& van Es, 2009) ont été récemment engagées.

Ria, Serres \& Leblanc (2010) proposent par exemple, au cœur de leur dispositif de formation, que les situations d'observation de pratiques enseignantes à partir d'enregistrements vidéo soient nécessairement complétées par une ou des situations d'observation en immersion dans les classes. Au cours de ces situations, les EN sont alors en effet invités « à analyser les situations professionnelles avec une plus grande acuité et à identifier de manière plus systématique les régularités des modalités d'agir » (Ria, Serres \& Leblanc, 2010, p. 114). Tout en relevant que l'observation à distance ou en immersion au sein des situations professionnelles " ne remplace aucunement l'expérience réelle mais la prépare " (Ria, Serres \& Leblanc, 2010 , p. 114), ces auteurs notent toutefois que ce couplage entre différents contextes de formation par l'observation peut être un moyen d'améliorer la pratique effective de classe des EN.

Dans le même ordre d'idées, notre étude (Gaudin \& Chaliès, 2011a) auprès d'EN d'éducation physique et sportive souligne l'importance de penser avec davantage de rigueur l'articulation entre les différentes situations de formation par l'observation, dont celles exploitant les enregistrements vidéo, et les situations de formation par l'intervention en classe. Les résultats de cette étude mettent plus précisément en exergue la nécessité d'une " contextualisation progressive " des situations de formation, avec tout d'abord une observation d'enregistrements vidéo à l'université, puis une observation directe en classe, et enfin un temps d'enseignement. Selon nous, la vidéo présente en effet l'avantage d'une gestion « contrôlable et contrôlée " des événements à visionner (comme la répétition d'une séquence). En ce sens, elle apparaît aux yeux des EN comme "facilitante » et donc plus intéressante en début de formation. Ce résultat est d'ailleurs complémentaire de ceux de l'étude menée par Sherin (2004), relevant que lorsqu'ils visionnent un enregistrement vidéo, les EN peuvent plus facilement être placés dans un «état d'esprit d'analyse ». Ce type de situation de formation leur offre en effet la possibilité de se détacher de contraintes normalement inhérentes à l'activité d'enseignement en classe, par exemple la nécessité de réagir immédiatement à toute perturbation, d'avoir un regard sur ce qui se passe dans l'ensemble de la classe, etc. De par leur caractère davantage situé (elles se déroulent en établissement scolaire et non à l'université), les situations d'observation d'un autre enseignant en classe ou d'eux-mêmes permettent aux EN de concrétiser et de finaliser les apprentissages introduits à l'université. Nous montrons également qu'il existe un " temps de latence " à respecter afin que les EN puissent exploiter ce qu'ils ont appris d'un type de situation à l'autre (l'observation d'enregistrements vidéo à l'université, l'observation en classe et lors de leur enseignement). Selon nous, ce temps de latence semble toutefois pouvoir être minimisé si les enregistrements vidéo montrés à l'université sont étudiés selon les contextes d'enseignement des EN.

Tout en gardant à l'esprit que dans les deux études citées préalablement (Gaudin \& Chaliès, 2011a ; Ria, Serres \& Leblanc, 2010), les contextes d'observation et d'intervention étaient des contextes de formation, leurs résultats permettent d'envisager les impacts des différentes situations d'observation, et notamment celle utilisant la vidéo, sur la pratique de classe des EN. D'autres travaux ont montré (sans complètement étayer leur propos par des données empiriques) que les EN redéploient en contexte d'intervention en classe des capacités d'observation et d'analyse développées initialement en contexte de formation par la vidéo (Borko, Koellner, Jacobs et al., 2011 ; Kersting, Givvin, Sotelo et al., 2010). Par exemple, les EN ayant antérieurement analysé les raisonnements des élèves au cours de situations d'observation utilisant des enregistrements vidéo parviennent semble- 
t-il plus facilement à répondre aux difficultés de compréhension des élèves lors de leur enseignement (Cohen, 2004).

Dans le même ordre d'idées, les résultats de l'étude de Sherin et van Es (2009) tendent à montrer que la "vision professionnelle " développée par les EN est ensuite exploitée en salle de classe. Les EN mobilisent en effet en apparence des stratégies similaires dans les deux contextes pour repérer et interpréter les raisonnements des élèves, même si, par exemple, un EN n'étudie pas le sens du raisonnement d'un élève de la même façon en regardant une vidéo ou dans la dynamique d'un questionnement en classe. Il semble en effet nécessaire d'engager de nouveaux travaux pour étudier davantage les effets des différents types de situation de formation sur la pratique de classe des EN (Seidel, Stürmer, Blomberg et al., 2011). Outre le fait d'asseoir un peu plus l'utilité de l'utilisation de la vidéo en formation, ces résultats permettraient de concourir à la construction de "dispositifs de formation hybrides " (Ria, 2010) au cœur desquels les activités d'observation et d'intervention se mêleraient continuellement (Gaudin \& Chaliès, 2011a). Tel que le notent Ria, Serres et Leblanc (2010), l'observation en formation ne peut en effet se suffire à elle-même, elle nécessite d'être pensée dans une relation " d'influence bidirectionnelle " (Sherin \& van Es, 2009) avec l'activité d'enseignement en classe qui tout à la fois l'introduit et la finalise.

\section{DU CARACTÈRE VIRTUEL DE L'ENSEIGNEMENT EN FORMATION À SA RÉALITÉ EN CLASSE}

L'ambition de cet article était, à partir d'un recensement de la littérature nationale et internationale, de circonscrire les différentes zones potentielles d'études quant à l'utilisation de la vidéo dans les situations de formation professionnelle des EN. Nous proposons finalement une poursuite des travaux de recherche dans quatre directions. Il semble tout d'abord important d'étudier plus en détail l'activité des EN impliqués dans ces situations. Le seul constat d'une difficulté avérée des EN à faire preuve de discernement lors de ce type de situation semble en effet à dépasser. En accord avec les résultats de l'étude de van Es et Sherin (2008), il apparaît plutôt nécessaire de chercher à appréhender la complexité de la construction de cette capacité professionnelle et d'examiner comment adapter les différentes modalités d'utilisation de la vidéo en fonction des besoins des EN. S'il existe de nombreuses modalités d'utilisation de la vidéo souvent ancrées dans des conceptions théoriques singulières, il semble en effet important d'interroger leur articulation (par exemple sous la forme d'un " continuum ") dans le but de professionnaliser les EN. Il conviendrait en outre d'approfondir l'étude des contenus proposés au sein de ce type de formation. De récents travaux appellent à un usage de contenus différenciés pour améliorer la formation. II reste néanmoins encore à étudier leurs possibles enchaînements. Enfin il apparaît nécessaire d'étudier les conséquences des situations de formation utilisant la vidéo sur la pratique de classe des EN. Inversement, de récentes études invitent à examiner comment cette dernière peut elle-même influencer en retour de nouvelles situations de formation. En s'efforçant de circonscrire ces différentes zones potentielles d'études, cet article met en évidence qu'elles sont traversées par une problématique commune relative aux circonstances dans lesquelles l'usage de la vidéo permet de préparer les EN à observer, analyser mais aussi intervenir en classe auprès de leurs élèves (Gaudin \& Chaliès, 2011b). II semble en effet important de développer des recherches permettant, d'une part, d'appréhender les différences existant nécessairement entre les circonstances 
de formation et d'intervention en classe et, d'autre part, de les minimiser. Ce n'est semble-t-il qu'à cette condition que les formateurs parviendront progressivement à faire de la vidéo un véritable trait d'union entre le caractère virtuel de l'enseignement en formation et sa réalité en contexte de classe.

Cyrille Gaudin

cyrille.gaudin@univ-tlse2.fr Université de Toulouse 2, EFTS et Institut universitaire de formation des maîtres de Midi-Pyrénées

Sébastien Chaliès sebastien.chalies@univ-tlse2.fr Université de Toulouse 2, EFTS et Institut universitaire de formation des maîtres de Midi-Pyrénées

\section{NOTES}

1 En ligne : http://tenue-de-classe.cndp.fr/ (consulté le 29 mars 2012).

2 En ligne : http://neo.ens-lyon.fr/neo (consulté le 29 mars 2012).

\section{BIBLIOGRAPHIE}

ANDRE T., SCHMIDT D., NONIS A., BUCK N. \& HALL S. (2000). "Preparing tomorrow's teachers today: Using videos of technology-using teachers to enhance preservice teachers' technology skills ". Communication présentée à la conference de la Society for Information Technology and Teacher Education international (SITE), San Diego.

BALL D. \& COHEN D. (1999). " Developing practice, developing practitioners: Toward a practice-based theory of professional education ». In G. Sykes \& L. DarlingHammond (dir.), Teaching as the learning profession: Handbook of policy and practice. San Francisco : Jossey-Bass, p. 3-32.

BENTON-KUPPER J. (2001). «The microteaching experience: Student perspectives ». Education, vol. 121, n० 4, p. 830-835.

BERTONE S., CHALIÈS S. \& CLOT Y. (2009). "Contribution d'une théorie de l'action à la conceptualisation et à l'évaluation des pratiques réflexives dans les dispositifs de formation initiale des enseignants ". Le Travail humain, vol. 72, n², p. 104-125.

BITTER G. \& HATFIELD M. (1994). «Training elementary mathematics teachers using interactive multimedia ". Educational Studies in Mathematics, vol. 26, no 4, p. 405-409.

BORKO H., JACOBS J., EITELJORG E. \& PITTMAN M. (2008). «Video as a tool for fostering productive discussions in mathematics professional development ". Teaching and Teacher Education, vol. 24, no 2, p. 417-436.

BORKO H., KOELLNER K., JACOBS J. \& SEAGO N. (2011). « Using video representations of teaching in practice-based professional development programs ». ZDM Mathematics Education, vol. 43, no 1, p. 175-187.

BROPHY J. (2004). Using video in teacher education. Oxford : Elsevier.

BRU M., PASTRÉ P. \& VINATIER I. (2007). "Les organisateurs de l'activité enseignante : perspectives croisées ". Recherche et formation, no 56, p. 95-108.

BRUNVAND S. (2010). "Best practices for producing video content for teacher education 》. Contemporary Issues in Technology and Teacher Education, vol. 10, no 2, p. 247-256.

CARTER K. (1999). "What is a case? What is not a case? » In M. Lundeberg, B. Levin \& H. Harrington (dir.), Who learns what from cases and how: The research base for teaching and learning with case. Mahwah: Lawrence Erlbaum, p. 165-175.

CASTRO A., CLARK K., JACOBS J. \& GIVVIN K. (2005). " Response to theory \& practice question: Using video to support teacher learning ". Association of Mathematics Teacher Educators Connections, vol. 14, no 3, p. 8-12.

CLARKE D. \& HOLLINGSWORTH H. (2000). "Seeing is understanding ". Journal of Staff Development, vol. 21 , no 4 , p. 40-43. 
COHEN S. (2004). Teachers' professional development and the elementary mathematics classroom: Bringing understanding to light. Mahwah: Lawrence Erlbaum.

COLESTOCK A. \& SHERIN M. (2009). « Teachers' sense-making strategies while watching video of mathematics instruction ". Journal of Technology and Teacher Education, vol. 17, no 1, p. 7-29.

COMMISSION DES COMMUNAUTÉS EUROPÉENNES (2007). “Améliorer la qualité des études et de la formation des enseignants ». Communication de la Commission au Conseil et au Parlement européen. Bruxelles : Commission des communautés européennes. En ligne : http://europa.eu/legislation_summaries/education_training_ youth/lifelong_learning/c11101_fr.htm (consulté le 29 mars 2012).

DELPOUX P. \& VEYRUNES P. (2010). «Analyse de l'activité de stagiaires lors de stages d'observation en formation des enseignants ". In Actes du congrès de l'Actualité de la recherche en éducation et en formation (AREF), Genève. En ligne : https://plone2.unige. $\mathrm{ch} /$ aref2010/communications-orales/premiers-auteurs-en-d/Analyse de lactivite.pdf/ view (consulté le 29 mars 2012).

DURAND M. (2008). «Un programme de recherche technologique en formation des adultes. Une approche énactive de l'activité humaine et l'accompagnement de son apprentissage-développement ". Éducation et didactique, vol. 2, n० 2, p. 69-93.

ERICKSON F. (2007). "Ways of seeing video: Toward a phenomenology of viewing minimally edited footage ». In R. Goldman, R. Pea, B. Barron \& S. Derry (dir.), Video research in the learning sciences. Mahwah: Erlbaum, p. 145-155.

ESCALIÉ G. \& CHALIÈS S. (2011). «Vers un usage européen du modèle des communautés de pratique en formation des enseignants ". Revue française de pédagogie, n० 174, p. 107-118.

FRANKE M., CARPENTER T., FENNEMA E., ANSELL E. \& BEHREND J. (1998). " Understanding teachers' self-sustaining, generative change in the context of professional development ». Teaching and Teacher Education, vol. 14, no 1, p. 67-80.

FREDERIKSEN J. (1992). "Learning to "see": Scoring video portfolios or beyond the hunter-gatherer in performance assessment ". Communication présentée à la réunion annuelle de l'American Educational Research Association, San Francisco.

GAUDIN C. \& CHALIÈS S. (2010). “ L'alphabétisation visuelle : vers la délimitation d'un nouveau champ de recherche en éducation et formation ". In Actes du congrès de I'Actualité de la recherche en éducation et en formation (AREF), Genève. En ligne : https://plone2.unige.ch/aref2010/communications-orales/premiers-auteurs-en-e/ Lalphabetisation.pdf/view (consulté le 29 mars 2012).

GAUDIN C. \& CHALIĖS S. (2011a). «Former par l'observation de pratiques professionnelles : précautions et pistes pour la construction de dispositifs de formation innovants ". In Actes du colloque des outils pour la formation, l'éducation et la prévention (OUFOREP), Nantes. En ligne : http://www.cren.univ-nantes.fr/98602891/0/fiche pagelibre/ (consulté le 29 mars 2012).

GAUDIN C. \& CHALIÈSS. (2011b). «L'usage de l'outil vidéo dans la formation professionnelle des enseignants novices par l'observation: circonstances dans lesquelles la virtualité permet de se préparer à la réalité ". Communication présentée au 14 e congrès de l'Association des chercheurs en activités physiques et sportives (ACAPS), Rennes.

GIRARD B. (1999). “Apprendre à observer ». Les cahiers EPS de l'académie de Nantes, no 19, p. 28-31.

GOLDMAN E. \& BARRON L. (1990). « Using hypermedia to improve the preparation of elementary teachers ». Journal of Teacher Education, vol. 41, n० 3, p. 21-31.

GOLDMAN R. (2007). "Video representations and the perspectivity framework: Epistemology, ethnography, evaluation, and ethics ». In R. Goldman, R. Pea, B. Barron \& S. Derry (dir.), Video research in the learning sciences. Mahwah : Lawrence Erlbaum, p. 3-38.

GOLDMAN R., PEA R., BARRON B. \& DERRY S. (2007). Video research in the learning sciences. Mahwah : Lawrence Erlbaum.

GOODWIN C. (1994). «Professional vision ». American Anthropologist, vol. 96, no 3, p. 606-633.

GROSSMAN P., WINEBURG S. \& WOOLWORTH S. (2001). « Toward a theory of teacher community". Teachers College Record, vol. 103, n० 6, p. 942-1012. 
HATCH T. \& GROSSMAN P. (2009). " Learning to look beyond the boundaries of representation: Using technology to examine teaching ". Journal of Teacher Education, vol. $60, \mathrm{n}^{\circ} 1, \mathrm{p} .70-85$.

HIEBERT J., GALLIMORE R. \& STIGLER J. (2002). « A knowledge base for the teaching profession: What would it look like and how can we get one? " Educational Researcher, vol. 31, n० 5, p. 3-15.

HUFFERD-ACKLES K., FUSON K. \& SHERIN M. (2004). " Describing levels and components of a math-talk learning community ». Journal for Research in Mathematics Education, vol. 35, n०2, p. 81-116.

JACOBS J. \& MORITA E. (2002). " Japanese and American teachers' evaluations of videotaped mathematics lessons ». Journal for Research in Mathematics Education, vol. 33, no 3, p. 154-175.

JACOBS V., LAMB L., PHILIPP R., SCHAPELLE B. \& BURKE A. (2007). "Professional noticing by elementary school teachers of mathematics ". Communication présentée à la réunion annuelle de l'American Educational Research Association, Chicago.

JACOBSON M. \& SPIRO R. (1995). « Hypertext learning environments, cognitive flexibility, and the transfer of complex knowledge: An empirical investigation ". Journal of Educational Computing Research, vol. 12, no 4, p. 301-333.

KALE U., WHITEHOUSE P. \& HAYES S. (2009). «The level of pedagogical content knowledge during video-based problem solving ". Communication présentée à la conférence annuelle de l'Association for Educational Communications and Technology, Teacher Education Division, Louisville.

KERSTING N., GIVVIN K., SOTELO F. \& STIGLER J. (2010). " Teachers' analyses of classroom video predict student learning: Further explorations of a novel measure of teacher knowledge ». Journal of Teacher Education, vol. 61, no 1, p. 172-181.

KLEINFELD J. (1988). Learning to think like a teacher: The study of cases. Fairbank: University of Alaska.

KOC M. (2011). « Let's make a movie: Investigating pre-service teachers' reflections on using video-recorded role playing cases in Turkey ". Teaching and Teacher Education, vol. 27, n०2, p. 95-106.

KOEHLER M. (2002). «Designing case-based hypermedia for developing understanding of children's mathematical reasoning ". Cognition and Instruction, vol. 20, no 2, p. 151195.

KOELLNER K., JACOBS J., BORKO H., SCHNEIDER C., PITTMAN M. \& EITELJORG E. (2007). «The problem-solving cycle: A model to support the development of teachers' professional knowledge ». Mathematical Thinking and Learning, vol. 9, n० 3, p. 273303.

KRAMMER K., RATZKA N., KLIEME E., LIPOWSKY F., PAULI C. \& REUSSER K. (2006). "Learning with classroom videos: conception and first results of an online teacher training program ». Zeitschrift für Didaktik der Mathematik, vol. 38, no 5, p. 422-432.

LAJOIE S. (2003). "Transitions and trajectories for studies of expertise ". Educational Researcher, vol. 32, no 8, p. 21-25.

LAVE J. \& WENGER E. (1991). Situated learning: Legitimate peripheral participation. Cambridge : Cambridge University Press.

LEBLANC S. (2009). “Des espaces de confrontation à l'expérience médiatisés par la vidéo : principes de conception, effets formatifs et projectifs ". In Actes du colloque international de l'association recherches et pratiques en didactique professionnelle: "L'expérience », Dijon. Disponible sur CD-ROM.

LEBLANC S. \& RIA L. (2010). “ Observatoire de l'évolution de la professionnalité enseignante et dispositifs de formation de simulation vidéo ». In G. Baillat, D. Nicot \& D. Ulma (dir.), La formation des enseignants en Europe. Approche comparative. Bruxelles : De Boeck, p. 205-213.

LE FEVRE D. (2004). «Designing for teacher learning: Videobased curriculum design ». In J. Brophy (dir.), Using video in teacher education. Oxford: Elsevier, p. 235-258.

LEINHARDT G., PUTNAM R., STEIN M. \& BAXTER J. (1991). « Where subject knowledge matters ». In P. Peterson, E. Fennema \& T. Carpenter (dir.), Advances in research on teaching. Greenwich : JAl Press, p. 87-113.

LEMKE J. (2007). «Video epistemology in- and outside the box: Traversing attentional spaces ». In R. Goldman, R. Pea, B. Barron \& S. Derry (dir.), Video research in the learning sciences. Mahwah: Lawrence Erlbaum, p. 39-51. 
MAYER R., MORENO R., BOIRE M. \& VAGGE S. (1999). " Maximizing constructivist learning from multimedia communicaitons by minimizing cognitive load ". Journal of Educational Psychology, vol. 91, no 4, p. 638-643.

MELOTH M. (2008). " The effects of video case analysis on preservice teachers' understanding of scaffolding ». In K. McFerrin, R. Weber, R. Carlsen \& D. Willis (dir.), Proceedings of Society for Information Technology \& Teacher Education International conference 2008. Chesapeake : AACE, p. 1488-1490.

MELOTH M., GOOD A. \& SUGAR W. (2008). «Review of research on the use of video cases to improve preservice and inservice teachers' knowledge and skills ". In K. McFerrin, R. Weber, R. Carlsen \& D. Willis (dir.), Proceedings of Society for Information Technology \& Teacher Education International conference 2008. Chesapeake : AACE, p. 940-942.

MERSETH K. (1996). "Cases and case methods in teacher education ». In J. Sikula, T. Buttery \& E. Guyton (dir.), Handbook of research in teacher education. New York: MacMillan, p. 46-62.

MINISTÈRE DE L'ENSEIGNEMENT SUPÉRIEUR ET DE LA RECHERCHE (2008). « Campagne d'habilitation de diplômes de master pour les étudiants se destinant aux métiers de l'enseignement. Rentrée universitaire 2009 ». Lettre du 17 octobre 2008 à Mesdames et messieurs les présidents d'université et directeurs d'établissement d'enseignement supérieur. Paris: Ministère de l'Enseignement supérieur et de la Recherche. En ligne : http://www.etatsgenerauxformationdesenseignants.fr/IMG/pdf/2008-10-17a.pdf (consulté le 29 mars 2012).

MINISTÈRE DE L'ENSEIGNEMENT SUPÉRIEUR ET DE LA RECHERCHE (2010). “ Mise en place des diplômes nationaux de master ouverts aux étudiants se destinant aux métiers de l'enseignement à la rentrée 2010 ». Circulaire $n^{\circ}$ 2009-1037 du 23 décembre 2009. Bulletin officiel du ministère de l'Enseignement supérieur et de la Recherche, no 1, 7 janvier 2010.

MORENO R., ABERCROMBIE S. \& HUSHMAN C. (2009). « Using virtual classroom cases as thinking tools in teacher education ». In I. Gibson R. Weber, K. McFerrin et al. (dir.), Proceedings of Society for Information Technology \& Teacher Education International conference 2009. Chesapeake : AACE, p. 2615-2622.

MOTTET G. (1996). "Du voir au faire. Le trajet de la vidéo-formation ». Recherche et formation, no 23, p. 29-54.

POSTIC M. (1977). Observation et formation des enseignants. Paris : PUF.

PUTNAM R. \& BORKO H. (2000). «What do new views of knowledge and thinking have to say about research on teacher learning? "Educational Researcher, vol. 29, n० 1, p. 4-15.

RIA L. (2010). «Néopass@ction. Une plate-forme de vidéo-formation ». Revue EPS, n 344, p. 19-21.

RIA L., SERRES G. \& LEBLANC S. (2010). “ De l'observation vidéo à I'observation in situ du travail enseignant en milieu difficile : étude des effets sur des professeurs stagiaires ». Revue suisse des sciences de l'éducation, vol. 32, no 1, p. 105-120.

RICHARDSON V. \& KILE R. (1999). «Learning from video cases ». In M. Lunderberg, B. Levin \& H. Harrington (dir.), Who learns what from cases and how? The research base for teaching and learning with cases. Mahwah: Lawrence Erlbaum, p. 121-136.

RODGERS C. (2002). "Seeing student learning: Teacher change and the role of reflection ». Harvard Educational Review, vol. 72, n², p. 230-253.

ROSAEN C., LUNDEBERG M., COOPER M., FRITZEN A. \& TERPSTRA M. (2008). « Noticing noticing: How does investigation of video records change how teachers reflect on their experiences? 》 Journal of Teacher Education, vol. 59, no 4, p. 347-360.

SANTAGATA R. (2009). «Designing video-based professional development for mathematics teachers in low-performing schools ». Journal of Teacher Education, vol. 60, n० 1, p. 38-51.

SANTAGATA R., ZANNONI C. \& STIGLER J. (2007). “The role of lesson analysis in preservice teacher education: An empirical investigation of teacher learning from a virtual video-based field experience ". Journal of Mathematics Teacher Education, vol. 10, no 2, p. 123-140.

SCHAEFFER J.-M. (1999). Pourquoi la fiction ? Paris : Éd. du Seuil.

SCHWINDT K. (2008). Lehrpersonen betrachten Unterricht: Kriterien für die kompetente Unterrichtswahrnehmung. Münster: Waxmann. 
SEIDEL T. \& PRENZEL M. (2007). "Wie Lehrpersonen Unterricht wahrnehmen und einschätzen: Erfassung pädagogisch-psychologischer Kompetenzen bei Lehrpersonen mit Hilfe von Videosequenzen ". Zeitschrift für Erziehungswissenschaft, vol. 8, no 1, p. 201-218.

SEIDEL T., STÜRMER K., BLOMBERG G., KOBARG M. \& SCHWINDT K. (2011). « Teacher learning from analysis of videotaped classroom situations: Does it make a difference whether teachers observe their own teaching or that of others? » Teaching and Teacher Education, vol. 27, no 2, p. 259-267.

SHERIN M. (2001). «Developing a professional vision of classroom events ". In T. Wood, B. Nelson \& J. Warfield (dir.), Beyond classical pedagogy: Teaching elementary school mathematics. Hillsdale : Lawrence Erlbaum, p. 75-93.

SHERIN M. (2004). "New perspectives on the role of video in teacher education ". In J. Brophy (dir.), Using video in teacher education. Oxford : Elsevier, p. 1-28.

SHERIN M. (2007). "The development of teachers' professional vision in video clubs ». In R. Goldman, R. Pea, B. Barron \& S. Derry (dir.), Video research in the learning sciences. Hillsdale : Lawrence Erlbaum, p. 383-395.

SHERIN M. \& van ES E. (2009). « Effects of video club participation on teachers' professional vision ". Journal of Teacher Education, vol. 60, no 1, p. 20-37.

SHERIN M. \& HAN S. (2004). "Teacher learning in the context of a video club ». Teaching and Teacher Education, vol. 20, n०2, p. 163-183.

SHULMAN L. (1992). "Towards a pedagogy of cases ". In J. Shulman (dir.), Case methods in teacher education. New York: Teachers College Press, p. 1-33.

SIMON M., TZUR R., HEINZ K., KINZEL M. \& SMITH M. (2000). “ Characterizing a perspective underlying the practice of mathematics teachers in transition ". Journal for Research in Mathematics Education, vol. 31, n० 5, p. 579-601.

SPIRO R., COLLINS B. \& RAMCHANDRAN A. (2007). "Reflections on a post-Gutenberg epistemology of video use in ill-structured domains: Fostering complex learning and cognitive flexibility ». In R. Goldman, R. Pea, B. Barron \& S. Derry (dir.), Video research in the learning sciences. Mahwah : Lawrence Erlbaum, p. 93-100.

STAR J. \& STRICKLAND S. (2008). « Learning to observe: Using video to improve preservice mathematics teachers' ability to notice ". Journal of Mathematics Teacher Education, vol. 11, n०2, p. 107-125.

SWELLER J. (1994). " Cognitive load theory, learning difficulty, and instructional design ». Learning and Instruction, vol. 4, no 4, p. 295-312.

SWELLER J. (2004). «Instructional design consequences of an analogy between evolution by natural selection and human cognitive architecture ». Instructional Science, vol. 32, no 1-2, p. 9-31.

TOCHON F. (1999). Video study groups: For education, professional development, and change. Madison : Atwood Publishing.

van ES E. \& SHERIN M. (2008). "Mathematics teachers' "learning to notice" in the context of a video club ». Teaching and Teacher Education, vol. 24, no 2, p. 244-276.

VARELA J. (1996). Invitation aux sciences cognitives. Paris : Éd. du Seuil.

VYGOTSKI L. (1978). Mind and society: The development of higher mental processes. Cambridge : Harvard University Press.

YADAV A. (2008). "What works for them? Preservice teachers' perceptions of their learning from video cases ». Action in Teacher Education, vol. 29, no 4, p. 27-38.

YAKURA E. (2009). " Learning to see: enhancing student learning through videotaped feedback ». College Teaching, vol. 57, n० 3, p. 177-183.

YAMAMOTO J. \& HICKS J. (2007). « Using digital movie and online discussion forum to facilitate microteaching for future secondary teachers ». In T. Bastiaens \& S. Carliner (dir.), Proceedings of world conference on e-learning in corporate, government, healthcare, and higher education 2007. Chesapeake: AACE, p. 6685-6688.

ZHANG M., LUNDEBERG M., KOEHLER M. \& EBERHARDT J. (2011). « Understanding affordances and challenges of three types of video for teacher professional development ». Teaching and Teacher Education, vol. 27, no 2, p. 454-462. 